\title{
A System of Simple Type Theory with Type Variables
}

\author{
Shôji Mamhara \\ Tokyo University of Education, Tokyo
}

The purpose of this paper is to give a system for simple type theory with type variables and to show that this system and Zermelo's set theory with individuals (= ur-elements) but without axiom of infinity can be reciprocally translated each other over an arbitrarily given first order theroy. In the following discussion, problems concerning the axiom of choice are not important.

\section{Type theory $T(\mathscr{T})$ over a given first order theory $\mathscr{T}$.}

1.1. 'Type'.

We use a type constant 0 and free type variables $\alpha, \beta, \ldots$ The type constant and each free type variable are said to be a type. If $\tau$ is a type, then also the expression $\tau+1$ is called a type. For exmaple, $\alpha+1$ and $(\alpha+1)+1$ and $((\alpha+1)+1)+1$ are types.

1.2. 'Formula'.

We use a binary relation symbol $\epsilon$ which is not used in the given first order theory $\mathscr{T}$. For every type $\tau$, we use free vriables $a_{\tau}, b_{\tau}, \ldots$, and bound variables $x_{\tau}$, $y_{\tau}, \ldots$, of type $\tau$. Finally, we use bound type variables $\xi, \eta, \ldots$

Our formulas in $T(\mathscr{T})$ are formed from simpler ones by means of the following operations:

1) the formation of the negation $\rceil \mathfrak{A}$ of a formula $\mathfrak{A}$;

2) the formations of the conjunction $\mathfrak{A} \wedge \mathfrak{B}$, the disjunction $\mathfrak{A} \vee \mathfrak{B}$, and the implciation $\mathfrak{A} \rightarrow \mathfrak{B}$ of formulas $\mathfrak{A}$ and $\mathfrak{B}$;

3) the quantifications of type $\tau$

$$
\forall x_{\tau} \mathfrak{A}\left(x_{\tau}\right) \text { and } \exists x_{\tau} \mathfrak{A}\left(x_{i}\right)
$$

of a formula $\mathfrak{A}\left(a_{\tau}\right)$ with respect to a free variable $a_{\tau}$ and a bound variable $x_{\tau}$;

4) the quantifications on types

$$
\forall \xi \mathfrak{A}(\xi) \text { and } \exists \xi \mathfrak{A}(\xi)
$$

of a formula $\mathfrak{X}(\alpha)$ with respect to a free type variable $\alpha$ and a bound type variable $\xi$, provided that the free type variable $\alpha$ appears in type of no free variable in $\mathfrak{A}(\alpha) \cdot *$

*) In my previous paper "Cut-elimination theorm concerning a formal system for ramified theory of types which admits quantifications on types" [this Annals, Vol. 2, pp. 5564 (1962) ], an analogous condition of the quantifications on ramified types must be added. 
A prime formula of $T(\mathscr{T})$ is a formula of the form $a \in b_{\tau}$ or a formula of the form $a=b_{\tau}$ or the result of replacing all free individual variables by free variables of type 0 throughout a prime formula of the theory $\mathscr{T}$.

1.3. Axioms and rules of inference.

As purely logical axioms and rules of inference we use ordinary ones for the classical predicate logic with equality. Non-logical axioms are following:

$\mathrm{T} 0$. The formulas obtained from axioms of the theory $\mathscr{T}$ by substituting free or bound variables of type 0 for all free or bound individual variables, respectively ("postulates" for the objects of type 0 );

T 1. $a_{\alpha} \notin b_{0}$;

T2. $a_{\alpha} \in b_{\beta} \rightarrow \exists x_{\beta}\left(a_{\alpha}=x_{\beta}\right)$;

T 3. $a_{\alpha} \in b+1 \rightarrow \exists x_{\beta}\left(a_{\alpha}=x_{\beta}\right)$;

T 4. $\exists \xi\left[\exists x_{\xi}\left(a_{\alpha}=x_{\xi}\right) \wedge \exists y_{\xi}\left(b_{\beta}=y_{\xi}\right)\right] \quad$ ("directedness of types");

T 5. $\left[M\left(a_{\alpha}\right) \wedge M\left(b_{\beta}\right) \wedge \forall \xi \forall x_{\xi}\left(x_{\xi} \in a_{\alpha} \rightleftarrows x_{\xi} \in b_{\beta}\right)\right] \rightarrow a_{\alpha}=b_{\beta}$, where $M\left(a_{\alpha}\right)$ means $\forall x_{0}\left(a_{\alpha} \neq x_{0}\right)$ and we read " $a_{\alpha}$ is a set" (axiom of "extensionality");

T 6. $\quad \exists y_{\alpha+1}\left[M\left(y_{\alpha+1}\right) \wedge \forall x_{\alpha}\left(x_{\alpha} \in y_{\alpha+1} \rightleftarrows \mathfrak{X}\left(x_{\alpha}\right)\right)\right]$ for each $\mathfrak{A}\left(x_{\alpha}\right)$ (axiom of "comprehension").

1.4. In this article, for the sake of convenience, we shall state some of formulas provable in $T(\mathscr{T})$ and give some definitions.

1.4 1. All formulas of the form

$$
\exists ! y_{\alpha+1}\left[M\left(y_{\alpha+1}\right) \wedge \forall x_{\alpha}\left(x_{\alpha} \in y_{a+1} \rightleftarrows \mathfrak{A}\left(x_{\alpha}\right)\right)\right]
$$

are provable, where $\exists ! y_{\beta} ₹\left(y_{\beta}\right)$ is an abbreviation of

$$
\exists y_{\beta}\left[\Re\left(y_{\beta}\right) \wedge \forall z_{\beta}\left(\Re\left(z_{\beta}\right) \rightarrow y_{\beta}=z_{\beta}\right)\right] \text {. }
$$

The existence of $y_{\alpha+1}$ is expressed in the axiom T6. The uniqueness of $y_{a+1}$ can be proved by the axioms $\mathrm{T} 3$ and $\mathrm{T} 5$.

1.411 . To represent the term expressed by

$$
\iota y_{\alpha+1}\left[M\left(y_{\alpha+1}\right) \wedge \forall x_{\alpha}\left(x_{\alpha} \in y_{\alpha+1} \rightleftarrows \mathfrak{Q}\left(x_{\alpha}\right)\right)\right]
$$

in usual notation, we shall introduce a symbol $\left\{x_{\alpha} \mid \mathfrak{A}\left(x_{\alpha}\right)\right\}$. By definition the relations

$$
\begin{gathered}
\exists y_{\alpha+1}\left(\left\{x_{\alpha} \mid \mathfrak{A}\left(x_{\alpha}\right)\right\}=y_{\alpha+1}\right), \\
M\left(\left\{x_{\alpha} \mid \mathfrak{A}\left(x_{\alpha}\right)\right\}\right)
\end{gathered}
$$

and

are identically true.

$$
\forall u_{\alpha}\left(u_{\alpha} \in\left\{x_{\alpha} \mid \mathfrak{A}\left(x_{\alpha}\right)\right\} \rightleftarrows \mathfrak{A}\left(u_{\alpha}\right)\right)
$$


1.412 . Definition of $D: \quad D=\left\{x_{0} \mid x_{0}=x_{0}\right\}$.

$D$ is the set of objects of type 0 .

1.4 12.1. $\exists \xi \exists x_{\xi}\left(x_{\xi} \in D\right)$.

$\left.1.412 .2 . \quad a_{\alpha} \in D \rightleftarrows\right\rceil M\left(a_{\alpha}\right), \quad M\left(a_{\alpha}\right) \rightleftarrows a_{\alpha} \notin D$.

These formulas can be easily proved by the axiom $\mathrm{T} 3$.

1.412 .3 . For all $\mathfrak{A}\left(x_{0}\right)$, the relations

and

$$
\forall x_{0} \mathfrak{A}\left(x_{0}\right) \rightleftarrows \forall \xi \forall x_{\xi}\left(x_{\xi} \in D \rightarrow \mathfrak{A}\left(x_{\xi}\right)\right)
$$

$$
\exists x_{0} \mathfrak{A}\left(x_{0}\right) \rightleftarrows \exists \xi \exists x_{\xi}\left(x_{\xi} \in D \wedge \mathfrak{A}\left(x_{\xi}\right)\right)
$$

are provable.

1.4 2. For each $\mathfrak{H}\left(x_{\mathfrak{a}}\right)$, the relation

$$
\begin{aligned}
\exists \zeta \forall \xi \forall x_{\xi}\left[\mathfrak{A}\left(x_{\xi}\right)\right. & \left.\rightarrow \exists z_{\zeta}\left(x_{\xi}=z_{\zeta}\right)\right] \\
\qquad & \rightarrow \exists \eta \exists y_{\eta}\left[M\left(y_{\eta}\right) \wedge \forall \xi \forall x_{\xi}\left(x_{\xi} \in y_{\eta} \rightleftarrows \mathfrak{A}\left(x_{\xi}\right)\right)\right]
\end{aligned}
$$

is provable.

Suppose that $\forall \xi \forall x_{\xi}\left[\mathfrak{A}\left(x_{\xi}\right) \rightarrow \exists z_{\gamma}\left(x_{\xi}=z_{\gamma}\right)\right]$ is true; let $b_{\gamma+1}=\left\{z_{\gamma} \mid \mathfrak{A}\left(z_{\gamma}\right)\right\}$; by the axiom $\mathrm{T} 3$ and by our supporsition on $\mathfrak{A}\left(x_{\xi}\right)$, the relation

$$
M\left(b_{\gamma+1}\right) \wedge \forall \xi \forall x_{\xi}\left(x_{\xi} \in b_{\gamma+1} \rightleftarrows \mathfrak{U}\left(x_{\xi}\right)\right)
$$

is true.

$1.421 . \quad \exists \eta \exists y_{\eta} \forall \xi \forall x_{\xi}\left[x_{\xi} \in y_{\eta} \rightleftarrows\left(x_{\xi}=a_{\alpha} \vee x_{\xi}=b_{\alpha}\right)\right]$.

Let $\gamma$ be a type such that $\exists x_{\gamma}\left(a_{\alpha}=x_{\gamma}\right) \wedge \exists y_{\gamma}\left(b_{\beta}=y_{\gamma}\right)$ is true (axion $\mathrm{T} 4$ ); then the relation

$$
\forall \xi \forall x_{\xi}\left[\left(x_{\xi}=a_{\alpha} \vee x_{\xi}=b_{\beta}\right) \rightarrow \exists z_{\gamma}\left(x_{\xi}=z_{\gamma}\right)\right]
$$

is true.

$1.422 . \quad \exists \eta \exists y_{\eta} \forall \xi \forall x_{\xi}\left[x_{\xi} \in y_{\eta} \rightarrow \exists \zeta \exists z_{\zeta}\left(x_{\xi} \in z_{\zeta} \in a_{\alpha}\right)\right]$.

Suppose that $b_{\beta} \in c_{\gamma} \in a_{\alpha}$ is true; then $\exists u_{\alpha}\left(c_{\gamma}=u_{\alpha}\right)$ (axiom T2), accordingly $\exists v_{\alpha}\left(b_{\beta}=v_{\alpha}\right)$ is ture (axiom T 2). Hence the formula

$$
\forall \xi \forall x_{\xi}\left[\exists \zeta \exists z_{\zeta}\left(x_{\xi} \in z_{\zeta} \in a_{\alpha}\right) \rightarrow \exists v_{\alpha}\left(x_{\xi}=v_{\alpha}\right)\right]
$$

is provable.

1.4 3. Definition of the relation symbol $\subset$ :

$$
a_{\alpha} \subset b_{\beta} \rightleftarrows\left[\forall \xi \forall x_{\xi}\left(x_{\xi} \in a_{\alpha} \rightarrow x_{\xi} \in b_{\beta}\right) \wedge M\left(a_{\alpha}\right) \wedge M\left(b_{\beta}\right)\right] \text {. }
$$

1.431 . $a_{\alpha} \subset b_{\beta} \rightarrow \exists y_{\beta+1}\left(a_{\alpha}=y_{\beta+1}\right)$.

Suppose that $a_{\alpha} \subset b_{\beta}$ is true; if $c_{\gamma} \in a_{\alpha}$ then $c_{\gamma} \in b_{\beta}$ and then $\exists y_{\beta}\left(c_{\gamma}=y_{\beta}\right.$ ) (axiom $\mathrm{T} 2)$; hence the relation

$$
\forall \xi \forall x_{\xi}\left[x_{\xi} \in a_{\alpha} \rightarrow \exists y_{\beta}\left(x_{\xi}=y_{\beta}\right)\right]
$$

is true. Let $d_{\beta+1}=\left\{y_{\beta} \mid y_{\beta} \in a_{\alpha}\right\}$; by the axioms $\mathrm{T} 3$ and $\mathrm{T} 5$, the relation $a_{\mathrm{a}}=d_{\beta+1}$ is true. 
1.432. $\exists \eta \exists y_{\eta} \forall \xi \forall x_{\xi}\left(x_{\xi} \in y_{\eta} \rightleftarrows x_{\xi} \subset a_{\alpha}\right)$

1.4 4. For each $\mathfrak{F}\left(x_{\xi}\right)$ and for each $a_{\alpha}$, the relation

$$
\exists \eta \exists y_{\eta}\left\{M\left(y_{\eta}\right) \wedge \forall \xi \forall x_{\xi}\left[x_{\xi} \in y_{\eta} \rightleftarrows\left(\mathfrak{Q}\left(x_{\xi}\right) \wedge x_{\xi} \in a_{\alpha}\right)\right]\right\}
$$

is provable.

Because $\forall \xi \forall x_{\xi}\left[\left(\mathfrak{A}\left(x_{\xi}\right) \wedge x_{\xi} \in a_{\alpha}\right) \rightarrow \exists z_{\alpha}\left(x_{\xi}=z_{\alpha}\right)\right]$ is provable (axiom T 2).

\section{$\S 2$. Zermelo's set theory $Z(\mathscr{T})$ over a given first order theory $\mathscr{T}$.}

$Z(\mathscr{T})$ is a theory formalzied in the usual first order perdicate logic with euqality. The non-logical constants of $Z(\mathscr{T})$ are those of the given first order theory $T$ and an object constant $D$ and a binary relation symbol $\epsilon$, the latters of which are not used in $\mathscr{T}$.

The non-logical axioms of $Z(\mathscr{T})$ are the following:

$\mathrm{Z} 0$. The formulas obtained from the non-logical axioms of $\mathscr{T}$ by replacing the parts of the form

with

$$
\forall x \mathfrak{F}(x) \text { or } \exists x \mathfrak{F}(x)
$$

$$
\forall x(x \in D \rightarrow \mathfrak{F}(x)) \text { or } \exists x(x \in D \wedge \mathfrak{F}(x)),
$$

respectively, where we assume without loss of generality that each axiom of $\mathscr{T}$ contains no free variable;

Z I. $\exists x(x \in D) \quad$ (existence of an individual);

Z2. $\forall x \forall y(y \in D \rightarrow x \notin y)$;

Z3. $\forall x \forall y\{[x \notin D \wedge y \notin D \wedge \forall z(z \in x \rightleftarrows z \in y)] \rightarrow x=y\}$

(axiom of extensionality);

Z4. $\forall x \forall y \exists z \forall u[u \in z \rightleftarrows(u=x \vee u=y)]$ (axiom of unordered pairs);

Z5. $\forall x \exists y \forall z[z \in y \rightleftarrows \exists u(z \in u \in x)] \quad$ (axiom of the sum set);

Z 6. $\forall x \exists y \forall z(z \in y \rightleftarrows z \subset x)$,

where $a \subset b$ means

$$
\forall x(x \in a \rightarrow x \in b) \wedge a \notin D \wedge b \notin D
$$

(axiom of the power set);

Z7. The formulas of the form

$$
\begin{gathered}
\forall x_{1} \ldots \forall x_{\downarrow} \forall x \exists y\left\{y \notin D \wedge \forall z\left[z \in y \rightleftarrows\left(\mathfrak{I}\left(z, x_{1}, \ldots, x_{\nu}\right) \wedge z \in x\right)\right]\right\}, \\
-134-
\end{gathered}
$$


which contain no free variables (axiom of Aussonderung).

Thововем. Let $\mathfrak{A}$ be a provable formula in $\boldsymbol{Z}(\mathscr{T})$ and contain no free variable. And let $\mathfrak{A}^{*}$ be the formula obtained from $\mathfrak{A}$ by replacing the parts of the form

with

$$
\forall x \mathfrak{F}(x) \text { or } \exists x \mathfrak{F}(x)
$$

$$
\forall \xi \forall x_{\xi} \mathfrak{F}\left(x_{\xi}\right) \text { or } \quad \exists \xi \exists x_{\xi} \mathfrak{F}\left(x_{\xi}\right)
$$

respectively, and by substituting $\left\{x_{0} \mid x_{0}=x_{0}\right\}$ for $D$ throughout $\mathfrak{A}$. Then $\mathfrak{X}^{*}$ is provable in $T(\mathscr{T})$.

It is sufficient to prove the theorem in only the cases where $\mathfrak{A}$ is a non-logical axiom of $\boldsymbol{Z}(\mathscr{T})$. In each of these cases, the assertion of the theorem is immediately obtained from axioms T0, T1, T5 and $1.411,1.412,1.412 .1,1.412 .2,1.412 .3$, $1.421,1.422,1.43,1.432,1.44$.

\section{§3. Interpretation of $T(\mathscr{T})$ in $Z(\mathscr{T})$.}

In the following, we give an interpretation of $T(\mathscr{T})$ in $Z(\mathscr{T})$. Then the considerations are all in the theory $Z(\mathscr{T})$.

3.1. $\exists y[y \notin D \wedge \forall x(x \notin y)]$.

By the axiom $\mathrm{Z} 7$, there exists a $y$ which makes

$$
y \notin D \wedge \forall x[x \in y \rightleftarrows(x \neq x \wedge x \in D)]
$$

true; for this $y$, the relation is true.

$$
\forall x(x \notin y)
$$

3.2. $\exists x \mathfrak{A}(x) \rightarrow[\forall x(x \in a \rightleftarrows \mathfrak{X}(x)) \rightarrow a \notin D]$.

By the axiom $Z 2$.

3.3. $\exists y \forall x(x \in y \rightleftarrows \mathfrak{A}(x)) \rightarrow \exists y[y \notin D \wedge \forall x(x \in y \rightleftarrows \forall(x))]$.

Let $\exists y \forall x(x \in y \rightleftarrows \mathfrak{A}(x))$ be ture; if $\exists x \mathfrak{A}(z)$ is true, by $3.2 \exists y[y \notin D \wedge \forall x(x \in y$ $\rightleftarrows \mathfrak{A}(x))]$ is ture; if $\exists x \mathfrak{A}(x)$ is false, by 3.1 we have the same result.

3.3 1. By virtue of 3.3 and the axiom $\mathrm{Z3}$, we can introduce the usual symbol $\{\mathbf{x} \mid \mathfrak{A}(x)\}$ such that the relations

and

$$
\{x \mid \mathfrak{A}(x)\} \notin D
$$

$$
a \in\{x \mid \mathfrak{A}(x)\} \rightleftarrows \mathfrak{A}(a)
$$

are ture, provided that the formula

$$
\exists y \forall x(x \in y \rightleftarrows \mathfrak{A}(x))
$$

is provable. 
3.4. Definition of 'Comp':

$$
\operatorname{Comp}(a) \rightleftarrows \forall x \forall y(x \in y \in a \rightarrow x \in a) \quad \text { (" } a \text { is complete"). }
$$

3.5. Definition of 'Type':

$$
\text { Type }(a) \rightleftarrows(\operatorname{Comp}(a) \wedge D \subset a) \quad \text { (" } a \text { is a type"). }
$$

3.5 1. Type $(D)$.

By the axiom $\mathrm{Z} 2$, Comp $(D)$ is true, then $D$ is a type.

3.5 2. Definition of 'the type 0'; $0=D$.

3.5 3. We use $\alpha, \beta, \ldots$ as free type variables and $\xi, \eta, \ldots$ as bound type variables.

3.531 . Comp $(\alpha)$

3.532 . $D \subset \alpha$.

3.6. Definition of ' $\alpha+1$ ':

$$
\alpha+1=\{x \mid x \in \alpha \vee x \subset \alpha\} .
$$

By the axioms $\mathrm{Z} 4, \mathrm{Z} 5, \mathrm{Z} 6$, we can prove the formula

$$
\exists y \forall x[x \in y \rightleftarrows(x \in \alpha \vee x \subset \alpha)] \text {. }
$$

3.6 1. Type $(a+1)$.

$D \subset a \subset a+1$ is clear. Then we prove only the fact that $a+1$ is complete. Suppose that $a \in b \in \alpha+1$ is true; $a \in b \in \alpha$ or $a \in b \subset \alpha$ is ture; if $a \in b \in \alpha$ ist rue, then $a \in a \subset a+1$, because $a$ is complete; if $a \in b \subset \alpha$ is ture, then we have directly $a \in \alpha \subset \alpha+1$.

3.7. For each type $\alpha$, we use free variables $a_{\alpha}, b_{\alpha}, \ldots$ and bound variables $x_{\alpha}, y_{\alpha} \ldots$ of type $\alpha$ as variables whose domain of variability is $\alpha$. By virtue of the axiom $Z 1$, we can see the fact that $\alpha$ is not empty (cf. 3.532 ), then the usual logical rules concerning the quantifiers $\forall x_{\alpha}$ and $\exists x_{\alpha}$ are all valid.

3.8. By the above-mentioned interpretation, the non-logical axioms of $T(\mathscr{T})$ are translated into the following formulas $3.80-3.86$ which are all provable in $Z(\mathscr{T})$.

3.8 0. The axioms of $\mathrm{Z} 0$. They are interpretations of axioms of $\mathrm{T} 0$.

3.8 1. $(a \in \alpha \wedge b \in D) \rightarrow a \notin b$.

By the axiom $\mathrm{Z} 2$.

3.8 2. $(a \in \alpha \wedge b \in \beta) \rightarrow[a \in b \rightarrow \exists x(x \in \beta \wedge a=x)]$.

Suppose that $b \in \beta$ and $a \in b$ are true; then $a \in \beta$ is true, because $\beta$ is complete.

3.8 3. $(a \in \alpha \wedge b \in \beta+1) \rightarrow[a \in b \rightarrow \exists x(x \in \beta \wedge a=x)]$.

Suppose that $b \in \beta+1$ is ture; $b \in \beta$ or $b \subset \beta$ is ture; if $a \in b \in \beta$, or if $a \in b \subset \beta$, $a \in \beta$ is ture.

3.84. $(a \in \alpha \wedge b \in \beta) \rightarrow \exists \xi[\exists x(x \in \xi \wedge a=x) \wedge \exists y(y \in \xi \wedge b=y)]$.

Let $\alpha \cup \beta=\{x \mid x \in \alpha \vee x \in \beta\}$ (cf. Z4 and Z5); $\alpha \cup \beta$ is complete and $D \subset \alpha \cup \beta$; then $\alpha \cup \beta$ is a type. If $a \in \alpha$ and $b \in \beta$, then $a \in \alpha \cup \beta$ and $b \in \alpha \cup \beta$. 
No. 4

3.85. $(a \in \alpha \wedge b \in \beta)$

$\rightarrow\{[a \notin D \wedge b \notin D \wedge \forall \xi \forall x(x \in \xi \rightarrow(x \in a \rightleftarrows x \in b))] \rightarrow a=b\}$.

This is regarded as an interpretation of the axiom $\mathrm{T} 5$, because

$$
\begin{aligned}
M\left(a_{\alpha}\right) & \rightleftarrows \forall x\left(x \in D \rightarrow a_{\alpha} \neq x\right) \\
& \rightleftarrows \forall x\left(a_{\alpha}=x \rightarrow x \notin D\right) \\
& \rightleftarrows a_{\alpha} \notin D .
\end{aligned}
$$

By the axiom $Z 3$, for the sake of proving the formula 3.85 , it is sufficient to prove the formula

$$
[a \in a \wedge b \in \beta \wedge \forall \xi \forall x(x \in \xi \rightarrow(x \in a \rightleftarrows x \in b))] \rightarrow \forall x(x \in a \rightleftarrows x \in b) .
$$

We shall prove this formula: suppose that $a \in \alpha$ and $b \in \beta$ and $\forall \xi \forall x(x \in \xi \rightarrow$ $(x \in a \rightleftarrows x \in b))$ are true;

$$
\forall x(x \in \alpha \cup \beta \rightarrow(x \in a \rightleftarrows x \in b))
$$

is true, because $\alpha \cup \beta$ is a type; if $x \in a$, then $x \in \alpha \subset \alpha \cup \beta$, accordingly $x \in b$ is true, and vice versa.

3.86. $\exists y\{y \in \alpha+1 \wedge y \notin D \wedge \forall x[x \in \alpha \rightarrow(x \in y \rightleftarrows \mathfrak{A}(x))]\}$

Let $b=\{x \mid \mathfrak{A}(x) \wedge x \in \alpha\}$ (cf. Z 7); then

$$
b \in D \quad \text { and } \quad \forall x[x \in \alpha \rightarrow(x \in b \rightleftarrows \mathfrak{A}(x))]
$$

are true. By definition $b \subset \alpha$ is true, then $b \in \alpha+1$.

Our translation is completed. 